Mathematical mindsets increase student motivation: Evidence from the EEG

Ian Daly, Jake Bourgaize, Alexei Vernitski

PII:

DOI:

S2211-9493(18)30034-6

Reference: TINE 108

To appear in:

Trends in Neuroscience and Education

Received date:

Revised date:

Accepted date:
16 July 2018

19 February 2019

27 February 2019

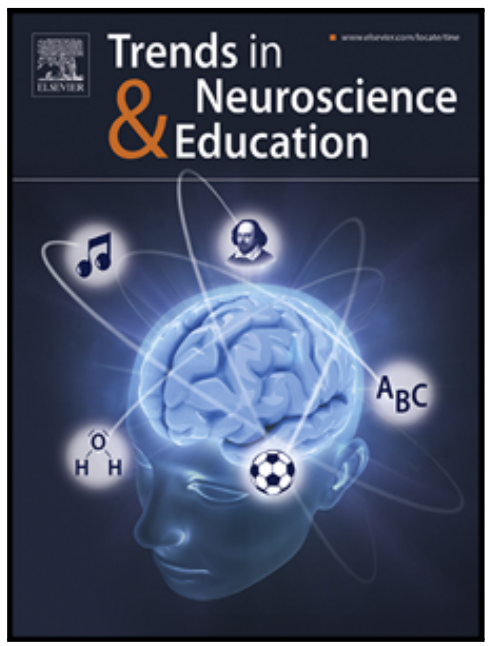

Please cite this article as: Ian Daly, Jake Bourgaize, Alexei Vernitski, Mathematical mindsets increase student motivation: Evidence from the EEG, Trends in Neuroscience and Education (2019), doi: https://doi.org/10.1016/j.tine.2019.02.005

This is a PDF file of an unedited manuscript that has been accepted for publication. As a service to our customers we are providing this early version of the manuscript. The manuscript will undergo copyediting, typesetting, and review of the resulting proof before it is published in its final form. Please note that during the production process errors may be discovered which could affect the content, and all legal disclaimers that apply to the journal pertain. 


\title{
Mathematical mindsets increase student motivation: Evidence from the EEG
}

\author{
Ian Daly ${ }^{\mathrm{a}}$, Jake Bourgaize ${ }^{\mathrm{b}}$, Alexei Vernitski ${ }^{\mathrm{c}}$ \\ ${ }^{a}$ Brain-Computer Interfacing and Neural Engineering Laboratory, School of Computer Science and \\ Electronic Engineering, University of Essex, Colchester, CO4 3SQ, UK ${ }^{b}$ Department of Psychology, \\ University of Essex, Colchester, CO4 3SQ, UK \\ ${ }^{c}$ Department of Mathematical Sciences, University of Essex, Colchester, CO4 3SQ, UK
}

\section{Abstract}

Mathematical mindset theory suggests learner motivation in mathematics may be increased by opening problems using a set of recommended ideas. However, very little evidence supports this theory.

We explore motivation through self-reports while learners attempt problems formulated according to mindset theory and standard problems. We also explore neural correlates of motivation and felt-affect while participants attempt the problems.

Notably, we do not tell participants what mindset theory is and instead simply investigate whether mindset problems affect reported motivation levels and neural correlates of motivation in learners.

We find significant increases in motivation for mindset problems compared to standard problems. We also find significant differences in brain activity in prefrontal EEG asymmetry between problems. This provides some of the first evidence that mathematical mindset theory increases motivation (even when participants are not aware of mindset theory), and that this change is reflected in brain activity of learners attempting mathematical problems.

Keywords:

Mathematical mindsets, EEG, motivation, prefrontal asymmetry

1. Introduction

Mathematics is one of the most important mental tools we possess as a species and is vital to the construction and smooth operation of modern civilization, underpinning everything from aerodynamics to zymology. Thus, it is necessary to ensure that as many members of society as possible have a firm grasp of the fundamentals of mathematics and that advanced mathematics are taught and understood effectively.

Effective mathematical education is, therefore, vital to the continued well-being of our society and is one of the cornerstones of modern civilization.

Mathematical cognition, the ability to use our mental processes to solve mathematical problems, is a learned process that is composed of many individual learned abilities [14]. These include simple learned abilities such as recognizing the numerosity of a set of objects, and recognising symbols such as ' + ' or ' $=$ ', through to relatively more advanced abilities such as being able to solve simultaneous equations or perform integration. 
Work in mathematical cognition has revealed that these learned abilities employ an extensive network of neural regions in our brain. For example, evaluating the numerosity of a set of objects involves neurons in the fundus of the intraparietal sulcus [39], while numerical cognition can involve perceptual, motor, mnemonic, and spatial functions [66]. Neuroimaging studies have shown numerous brain regions to be involved in all these functions, for example, the motor cortex is active during motor function processing related to mathematical cognition [66], while the intraparietal sulcus, left angular gyrus area, and the bilaterial posterior superior system support processing of numbers [24]. Additionally, a common feature of all these functions is activity in the parietal lobes which is observed during almost all mathematical and numerical tasks $[51,41]$.

Furthermore, the mental workload involved in mathematical cognition is known to linearly relate to the absolute level of neural activity in the cortex [3]. Although the topology of that change in neural activity across the cortex is known/to be focused on specific regions of the brain, including the right prefrontal cortex, which is known to exhibit increases in neural activity (as measured by EEG) when mental workload increases [29].

Efforts to improve mathematical education, to better train learners in mathematical cognition, often focus on the didactics of mathematics i.e. on the pedagogy or methods employed to teach mathematics [8]. Mathematical education methods span a range of approaches, including, but not limited to, classical education (based on deductive reasoning), computer-based approaches, worked exercises and problem solving to educate via reinforcement, and rote learning [4]. Modern contemporary mathematical education methods typically make use of a range of these techniques, with each method chosen based on the needs and abilities of the learner and the particular numerical abilities to be taught [4],

However, in order to effectively teach mathematical abilities (or any subject) willing and engaged learners are highly desirable $[60,50]$. The more motivated and engaged the learner, the more likely the material will be learned successfully, and the less time it will take learners to understand new material [61]. Thus, it may be said that, in general, learner engagement and motivation underpin and drive the success or failure, of all mathematical didactic methods.

Consequently, modern approaches to mathematical didactics increasingly aim to engage the/learner in self-motivated learning $[50,61]$. Approaches to this include the careful design of homework given to students [25] and attempts to foster value beliefs in the relevance of mathematics in adult life in students [31]. However, despite a number of intervention studies, there is no generally agreed upon best method to engage and motivate learners in mathematical learning [43].

Researchers studying motivation in mathematical learners have considered motivation as something aligned with the learners' life goals and values (such as 'achieving mastery'). Motivation, understood in this way, consists of many parts and evolves over a long time $[59,55,17,44,16,13,36,63]$. Motivation is not the same in different gender groups $[58,65]$ and special activities, such as providing a reward system, may be used in class to attempt to improve motivation [1]. 
The neural basis for motivation is based upon the neurotransmitter dopamine, which, when transmitted through the mesolimbic reward pathway acts to regulate motivation and reward responses [42]. The exact mechanism by which dopamine modulates motivation is unclear [42]. However, numerous neuroimaging studies have shown significant changes in activity in the emotion response network related to changes in dopamine levels and behavioral observations of changes in motivation [42].

The emotion response network includes the anterior cingulate cortex, the amygdala, and interactions between the midbrain regions and the lateral frontal cortex, which have all been shown, in neuroimaging studies, to contain activity that correlates with motivation $[37,6]$. The most easily observable parts of the emotion response network are the parts that reside in the cortex, as these may readily be observed via neurophysiological measurement modalities that can be applied relatively easily during ecologically relevant tasks, such as the electroencephalogram (EEG). The prefrontal cortex is of particular interest. Changes in EEG bandpower in the prefrontal cortex have been shown to be modulated by affect, approach-withdrawal responses to stimuli, and motivation [18]. Specifically, a growing number of studies have reported changes in hemispheric asymmetry (the relative difference) in EEG activity in the left and right hemispheres) in EEG activity within specific frequency bands in response to changes in motivation. More motivating events and tasks have been reported to produce greater magnitude EEG alpha and beta bandpower in the left prefrontal cortex in response to affective and motivating stimuli $[62,52]$ and during changes in motivation related to a task [57]. This is encapsulated in the "hemispheric valence hypothesis', which states that the approach response to stimuli (such as increased motivation) is processed more in the left hemisphere and the withdrawal response is processed more in the right hemisphere [52].

One promising approach to pedagogy that aims to increase learner motivation is mindset theory. This was developed by Carol Dweck and her collaborators, and studies what motivates and demotivates learners [27]. Mindset theory postulates that learners hold one of two views about their abilities. Learners either believe they have a 'fixed mindset' in which their ability to perform at a particular task is fixed and innate, or a 'growth mindset' in which their ability can be improved with practice [26].

Under mindset theory, the particular mindset a learner believes they possess is thought to affect their motivation in a learning environment [28]. For example, an individual who has a fixed mindset may believe they are innately bad at learning mathematics. Consequently, they may avoid putting effort into tasks that involve mathematical thinking. By contrast, an individual with a growth mindset is thought to be more likely to be motivated to engage with learning that focuses on areas of perceived weakness as this will allow them to improve their skills and advance their knowledge [32, 2].

Many of the challenges to mathematical education, such as 'mathematics anxiety' in learners, may be thought of in terms of fixed mindsets and growth mindsets. Following Carol Dweck's work, Jo Boaler and her collaborators studied which specific changes in mathematical education courses can increase learners' motivation $[11,10$, 9]. 
Jo Boaler's mathematical mindset (MM) theory is an approach to teaching mathematics that seeks to encourage a growth mindset in learners. Whereas some recommendations of Boaler and collaborators are related to the global structure of a mathematical course (such as how much homework to have), others specifically describe what features of a particular problem presented to students in class may increase their motivation. It is known, from Dweck's experiments, that the right (or wrong) kind of activities can have an immediate influence on mindsets [28]. Individual activities such as mathematical problems can affect the learners' mindsets. For example, activities should be intrinsically motivating (i.e. interesting) to students. They should also be designed to allow some degree of choice and autonomy by students [48].

There is sociological research studying the impact of MM theory, for example, [12]. On the other hand, there is some neurophysiological research studying observed differences in neural activity between persons with a growth mindset and a fixed mindset. For example, individuals with a growth mindset have been reported to have a higher Pe (error positivity) waveform response in their EEG than individuals without a growth mindset [46] (also see the review [48]).

However, to date there is very little evidence to suggest that MM problems actually encourage students to be more motivated during the activity. There is also very little evidence exploring the neural mechanisms underlying changes in motivation levels that underpin the theory of MM based pedagogy [48]. Furthermore, the majority of existing studies explicitly make participants aware of mindset theory. This approach does not explicitly test whether individual problems structured according to MM theory can, by themselves, affect motivation, and that may encourage some participants to view MM problems in a more favourable light, leading to potentially biased results.

Therefore, we set out to understand whether problems formulated according to MM theory affect learner motivation. We explore this through an investigation into the underlying neural mechanisms underpinning MM interventions [48]. Our study uses psychological and neurophysiological methods to examine the immediate reaction to MM problems as the participants attempt a random selection of standard mathematical problems and MM problems. Thus, we set out to investigate whether mathematical problems that have been structured as MM problems increase learner motivation via a combination of behavioural and neurophysiological measures.

Our research exists in the context of the study of motivation of students studying mathematics; this is why it is important to explain our approach to motivation. In this paper we consider short-term levels of motivation, which is the willingness to engage with a given learning activity. Our approach follows that of Carol Dweck [27]; she established that motivation during a learning activity can be very quickly affected by how this activity or the previous activity was presented to the learner. Accordingly, in our experiments we present a mathematical problem to a participant in one or another way and measure the short-term effect produced by the problem while the participant is attempting it (using the EEG) and after the participant has finished working on it (using questions about the participant's level of motivation). Via MM theory, our understanding of motivation can be said to have something in common with some of the aspects of long-term motivation in [59], including enjoyment, self-confidence and 
willingness to take risks, since these are the qualities that a growth mindset is believed to promote.

We employ a combination of Psychological test batteries to investigate changes in participant self-reports of affect and motivation as learners attempt MM problems and standard equivalent problems which have not been structured according to MM theory. Additionally, motivated by recent efforts to explore the neuroscience of mindsets [48], we explore neural correlates of motivation in the EEG as participants tackle different types of mathematical problems presented traditionally and presented in a way that is intended to promote a growth mindset. We make the following hypotheses.

1. Participants will report that they find $\mathrm{MM}$ problems more motivating than standard problems.

2. Prefrontal EEG asymmetry, a neural response to changes in motivation, will differ significantly between MM problems and standard problems.

\section{Methods}

\subsection{Participants}

Twenty three participants (13 female) were recruited to our study from the undergraduate student population of the University of Essex. Participants were recruited on the basis that they were currently enrolled on their first or second year of undergraduate study in the department of Mathematical Sciences via direct emails to the student body. Participants were paid for their time at a rate of $f 20.00$ (GBP). The mean age of the participants was $20( \pm 0.74)$ years. All participants were right handed.

Ethical permission for this study was granted by the institutional review board of the University of Essex.

\subsection{Experiment design}

Participants were sat in front of a computer screen with a keyboard placed on their right to answer Likert scale questions and progress the experiment, as well as a pen and paper, placed in front of them, to show workings out and answers to mathematical problems. Once the experiment began, participants were presented with three statements, which provided a measure of their motivation and their current affective states prior to attempting to solve each of the mathematical problems.

Specifically, participants were first asked to report their current felt levels of motivation via three statements using 5 point Likert scales. The motivation questions were adapted from the 'Program for international student assessment' [38]. Specifically, the sentences they were presented with were, in sequence, "I am strongly motivated to solve the problem.", "I intend to put in a good effort solving this problem." and "Doing well at this problem means a lot to me.". Participants were also asked to report their current felt affective states via self-assessment manikins for valence and arousal [45]. Following this, participants were presented with either a standard or a MM style mathematical problem to solve. Participants were given a maximum of 5 minutes to solve the presented problem but could progress the 
experiment at any time before this time elapsed with the press of the space-bar on the keyboard once they had finished their attempt to solve the problem.

Finally, participants were asked to report their motivation and affective states again after the problems had been presented. This was also done using the selfassessment manikin and a 5 point Likert scales using similar statements to before problem presentation, but in the past tense. These measures were employed in order to assess how motivated participants felt as a result of attempting to solve the problem. Specifically, the following motivation assessment statements were presented, "I was strongly motivated to solve the problem.", "I put in a good effort solving this problem." and "Doing well at this problem meant a lot to me.". After each trial participants were given a 2.5 second inter-stimulus interval rest period. This procedure was repeated until the participants had completed 10 problems.

A within-subjects study design is used and problems were presented in a pseudorandomised order to each participant after being drawn from a pool of 10 standard and $10 \mathrm{MM}$ problems. For each participant, problems were uniformly drawn without replacement from the problem pools. Out of each pair of problems (one formulated in the standard way, and the other as a MM problem) only one was presented to each participant. Participants were not informed, prior to the experiment, about what $\mathrm{MM}$ theory is. Over all participants an equal number of standard and $\mathrm{MM}$ problems were presented.

\subsection{Problems}

Pairs of questions were prepared from several areas of mathematics which firstyear undergraduate students should be familiar with (all questions are included below in the Appendix). In each pair of questions, one was written in what can be considered a standard style, for example, like in $[5,20]$, and one was written with small changes made according to recommendations of MM theory.

In general, the MM recommendations for writing a mathematical problem can be briefly summarised as follows (see Chapter 5 in [11] for both this list and a more detailed discussion).

- Open up the task so that there are multiple methods, pathways, and representations.

- Include inquiry opportunities.

- Ask the problem before teaching the method.

- Add a visual component and ask students how they see the mathematics.

- Extend the task to make it lower floor and higher ceiling.

- Ask students to convince and reason; be skeptical.

Specifically, the changes were applied as follows: 
Problem pair 1. The MM version of the question explicitly lists all elements to be used in the answer; the student needs to connect them in the right order, like a jigsaw puzzle.

Pair 2 and Pair 10. The MM version conceals the fact that the question, effectively, asks the student to do a very standard calculation (solve a standard system of two linear equations or multiply two $2 \times 2$ matrices).

Pair 3 and Pair 5. The MM version asks the same question, but as a geometric question (find the position of a point, or the distance between points) instead of an algebraic question. In the MM version, the student needs to guess what formula to use, whereas in the standard version the formula is given explicitly in the question.

Pair 4. The MM version invites the student to guess a solution of a differential equation using an example (a solution of another, simpler differential equation), whereas the standard version asks the student to find the same answer by solving an equation explicitly.

Pair 6. The standard version instructs the student to use a particular method (the derivative), but the MM version omits this recommendation.

Pair 7. The question asks the student to find a particular solution of a differential equation. The standard version explicitly includes the general solution, but the MM version leaves it up to the student to guess it.

Pair 8 and Pair 9. The standard version includes the formula to use, but the MM version omits the formula, so the student will need to come up with their own way of finding the answer.

\section{4. $E E G$}

We investigated neural correlates of the approach - withdrawal response and motivation [15] to the different types of problem. In order to do this, EEG was recorded from 32 electrodes positioned according to the international 10/20 system for electrode placement via a Biosemi ActiveTwo system. Impedances on all channels were kept below $10 \mathrm{k} \Omega$ for the duration of the recording.

As a first pre-processing step, the EEG was cleaned of physiological artifacts via the automated EEG artifact removal method 'Fully automated online artifact removal' ('FORCe'; [22]). This method uses a combination of independent component analysis (ICA) and Wavelet transforms to identify and remove physiological artifact components from the EEG signals and has previously been validated in studies with both healthy participants and participants with movement impairments [22]. The EEG was then rereferenced to a common average reference (CAR) scheme to reduce the effects of localized noise on the signals. Finally, it was filtered between $1-45 \mathrm{~Hz}$ using a 2 nd order Butterworth filter and visually spot-checked by an experienced EEG researcher (author ID, 10+ years experience) to ensure it was free of physiological artifacts. Additionally, 
all other data recorded from all participants, including behavioural responses, were visually inspected for recording errors and artifacts.

\subsection{Analysis of participant reports}

Participants self-reports of their affective states and levels of motivation were inspected before and after they attempt to solve each problem. The change in a participant's level of reported motivation was then compared between problem types (standard or MM problems) over all participants on a per problem basis and in aggregate over all problems. The significance of the change in motivation reported by participants after completing each of the two types of problems was evaluated via a student's $t$-test.

\subsection{Analysis of EEG}

Laplacian derivations were extracted from the EEG to describe the activity in key frequency bands over the left and right prefrontal cortices. Specifically, Laplacian derivations were extracted from regions of the left and right prefrontal cortex centered on channels F3 and F4 within the alpha $(8-13 \mathrm{~Hz})$, beta $(13-25 \mathrm{~Hz})$, and gamma $(25+\mathrm{Hz})$ frequency bands. These choices were based on previous reports of observed neural correlates of affect and motivation in these bands [47, 64, 21].

Laplacian derivations were calculated as the band-power on channels F3 and F4 minus the mean band-power of the surrounding channels. For channel F3 the surrounding channels were FP1, F7, Fz, and C3, while for channel F4 the surrounding channels were FP2, F8, Fz, and C4.

Asymmetry was then defined as the left laplacian derivation minus the right laplacian derivation. The time series of asymmetry values was than segmented into a set of trials focused on the first 30 s of EEG recorded, when the participants began to attempt to solve the presented problems. We suggest that this time period best captures activity in the participants' brains as they first approach the problem and begin to attempt to solve it. These trials were extracted from all attempts by participants to solve the problems, regardless of trial type or participant success at the task. Each trial was than base-line corrected to normalize across trials to participants current background EEG bandpower magnitudes. Specifically, mean baseline asymmetry values from the $2 \mathrm{~s}$ prior to the start of each trial were subtracted from each trial. Mean asymmetry values across trials were then compared between conditions (standard problems vs. MM problems) across all participants for each problem type and for all problems. An ANOVA and student's $t$-tests were used to statistically evaluate the differences in results.

To further verify the validity of EEG asymmetry as an index of motivation we also calculate the correlation between the EEG asymmetry in each frequency band and participant reports of their change in motivation after attempting each problem (the difference in their reported motivations before and after attempting each problem). We anticipate that, if the prefrontal EEG asymmetry is a reliable index of motivation, we should observe significant correlations. 


\subsection{Task performance effects}

We investigated effects of problem type on task performance and whether formulating problems according to MM theory was more beneficial for some groups of students than others. Specifically, we wished to see if MM theory was more motivating for 'weaker' students.

Here, when we state 'stronger' or 'weaker' student, we refer to their performance when solving the standard problems. Specifically, we looked at how many standard problems each participant got correct and from this estimated the probability that a given participant will get a standard problem correct. We suggest this provides a crude, but workable, measure of whether a participant is a 'strong' or 'weak' student in the context of our experiment.

We then measured the correlations between the probability a participant will get a standard mathematical problem correct and their change in motivation levels between standard problems and MM problems (as measured by both participant selfreports and neural correlates of motivation). We suggest that, if MM problems are more motivating for 'weaker' students (or indeed for 'stronger' students) there will be a correlation between performance and change in motivation between standard problems and MM problems.

3. Results

\subsection{Participants}

A total of 7 participants were removed from the dataset at the initial inspection stage due to a combination of EEG artifacts and technical errors in recording behavioral responses. Thus, 16 participants remained in the dataset.

\subsection{Self-reports}

The changes in motivation levels reported by participants after completing each mathematical problem may be evaluated by considering each motivation test question in isolation, or by considering all 3 questions in aggregate. When reported changes in motivation, over all participants, are considered for each motivation test question individually, a greater reduction in motivation is reported for standard problems compared to $\mathrm{MM}$ for all three questions. However, these differences were not observed to be significantly different ( $t$-test, $p>0.05)$. These results are illustrated in Figure 1.
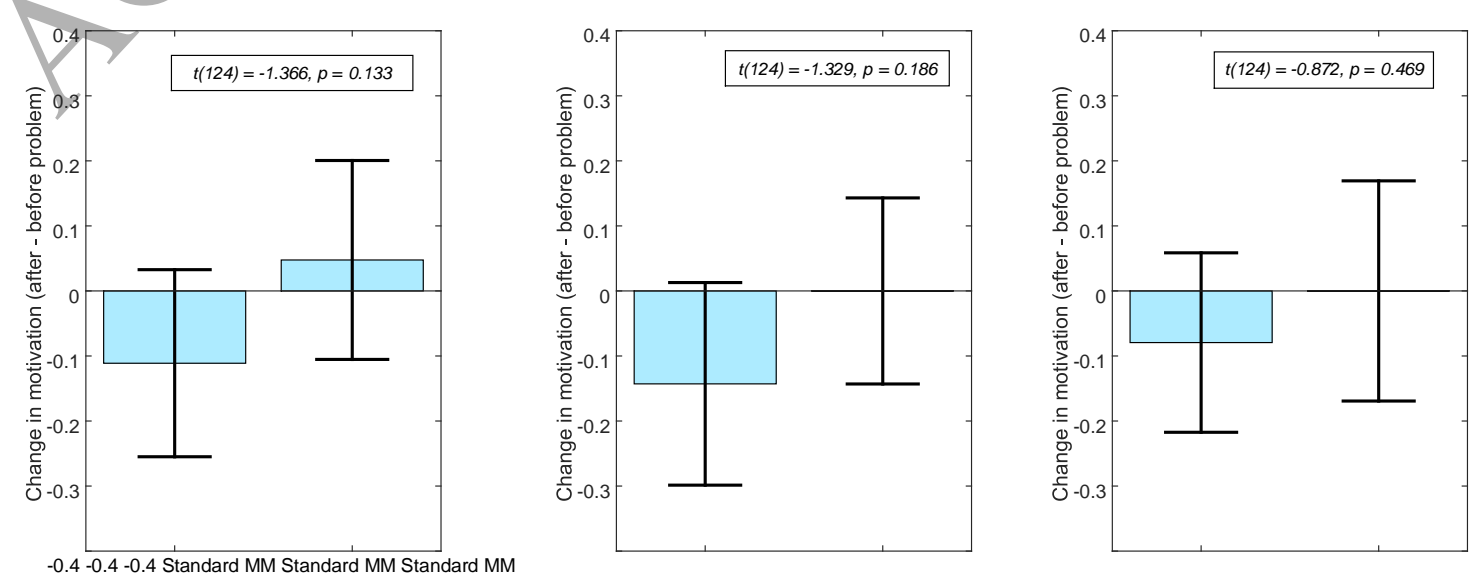

(a) Motivation test question 1
(b) Motivation test question 2
(c) Motivation test question 3

Figure 1: Change in motivation reported over all participants after attempting each type of problem (standard and MM standard problems). Change is reported as the difference in motivation in response to each of the questions after attempting the problem minus the reported motivation before attempting the problem. The mean over all participants and problems of each type (either standard or MM) is reported. Error bars indicate the confidence intervals. Significance testing is performed with a Student's $t$-test.

However, when participants' reports of their motivation are considered in aggregate (i.e. the responses to all three motivation test batteries are combined), a significant difference in change in motivation is observed between standard problems and $\mathrm{MM}$ problems $(t(376)=-2.063, p=0.039)$. Therefore, we may conclude that participants report a reduction in motivation when attempting standard problems, while for MM problems there is a marginal increase in motivation. This result is illustrated in Figure 2.

Participant self-reports of their felt affective states (valence and arousal) were also investigated in the same way. However, no significant changes in reported affect were observed in the case of valence $(t(124)=1.356, p=0.177)$ or arousal $(t(124)=0.798$, $p=0.426)$.

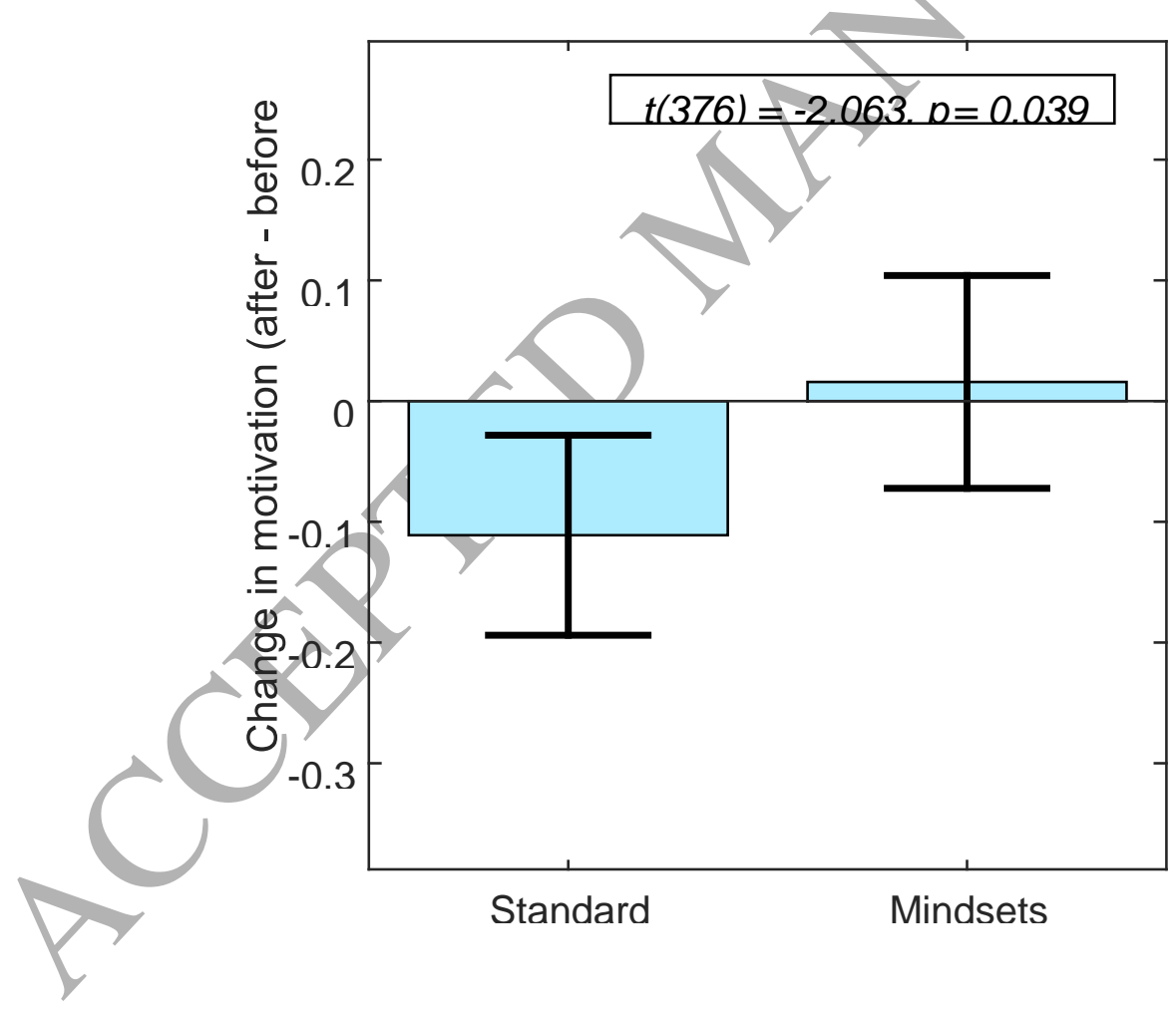

Figure 2: Change in motivation reported over all participants after attempting each type of problem (standard and MM problems). Change is reported as the difference in motivation in response to an aggregate of all the questions after attempting the problem minus the reported motivation before attempting the problem. The mean over all participants and problems of each type is reported. Error bars indicate the confidence intervals. Significance testing is performed with a Student's $t$-test. 
We also investigated how participant self-reports differ between MM and standard problems for each problem individually. The changes in motivation levels reported by all participants for each individual problem are illustrated in Figure 3.

It may be noted that problems 2, 5, and 10 all exhibit significantly greater increases in reported motivation compared to standard problems and that several of the other problems (4, 6, and 9) also exhibit non-significant increases in motivation when they are presented as MM problems. However, after application of HolmBonferroni multiple comparisons correction none of these results remained significant at $p<0.05$, suggesting that any differences on a problem-by-problem basis are only marginal and that one should not draw too strong conclusions from these individual problem based results. Note, when participants attempted problem 3 formulated as a standard problem they did not report any changes in motivation.

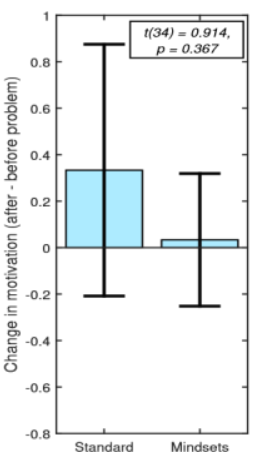

(a) Problem 1

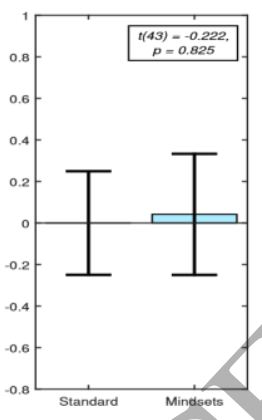

(f) Problem 6

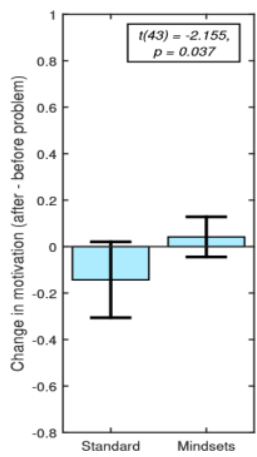

(b) Problem 2



(g) Problem 7

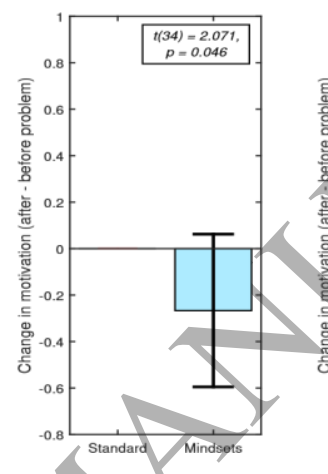

(c) Problem 3

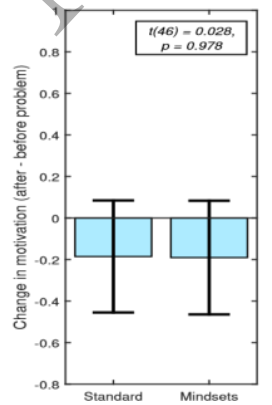

(h) Problem 8

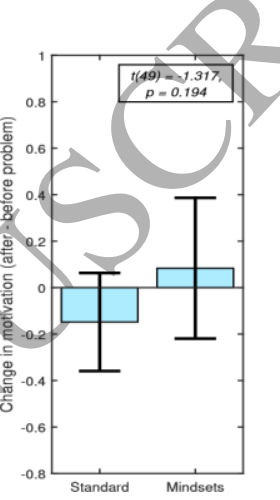

(d) Problem 4

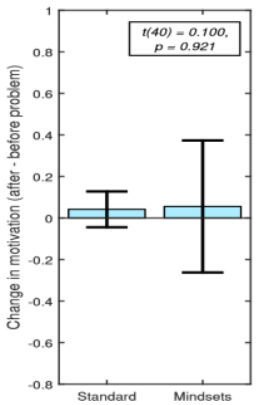

(i) Problem 9

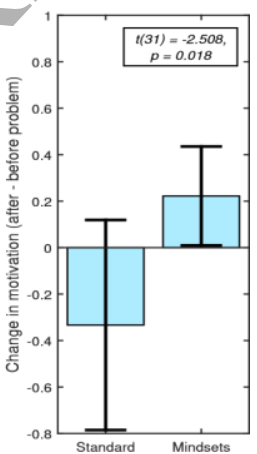

(e) Problem 5

Figure 3: Change in motivation reported over all participants after attempting each type of problem (standard and MM problems) for each individual problem. Change is reported as the difference in motivation in response to each of the questions after attempting the problem minus the reported motivation before attempting the problem. The mean over all participants and problems of each type is reported. Error bars indicate the confidence intervals. Significance testing is performed with a Student's $t$-test.

\subsection{Task performance}

The difficulty of each of the mathematical problems is also an important consideration when evaluating the results. Specifically, due to the nature of the way MM problems are presented, they may differ significantly in difficulty to the standard problems. 
Difficulty is a subjective measure of the challenge a given problem presents to each person and, therefore, is most accurately measured by direct questioning of each participant. Unfortunately, direct querying of task difficulty was not carried out in our study. However, indirect measures of task difficulty may be performed by inspecting individual performance at each problem type.

Specifically, the accuracy of each participant at answering questions of each problem type was measured by first marking the answers given by each participant to each question via a binary mark scheme ( $0=$ wrong, $1=$ correct). The accuracy of each participant at answering questions from each problem type was then measured by dividing the number of correct answers for each problem type by the total Problem type Mean accuracy ( \pm STD)

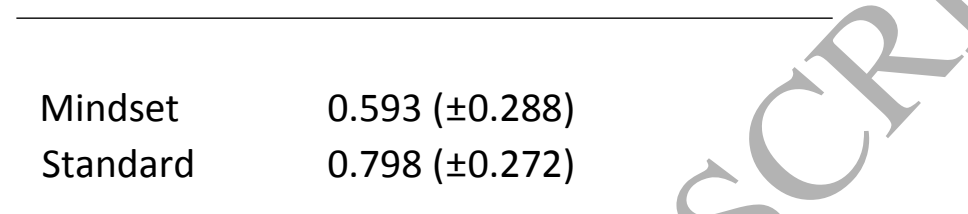

Table 1: Accuracy of the participants at answering each type of mathematical problem.

number of attempts at each problem type. The results are listed in Table 1.

A significant difference $(t(30)=-2.062, p=0.048)$ is observed between MM problems and standard problems. From this we may conclude that participants were significantly more accurate in getting the correct answers when the problems were presented in a standard manner than when the problems were presented via the MM framework. (Note that this does not mean that MM problems are always more challenging than standard problems in practice. The difference seen in this experiment is the result of preparing pairs of problems which cover exactly the same topics and share the same answers.)

\section{4. $E E G$}

EEG prefrontal asymmetry was compared between standard problems and MM problems in each of the key frequency bands of interest. We first apply an ANOVA with factors 'problem type' ('standard' or 'MM') and 'frequency band' ('alpha', 'beta', or 'gamma').

The only significant effect we find is the effect of 'problem type' $(F(1,458)=$ $19.290, p<0.001$, Partial Eta Squared $=0.033$ ). The effect of 'frequency band' was not significant $(F(2,458)=0.620, p=0.537$, Partial Eta Squared $=0.002)$, and no significant interactions were observed $(F(2,458)=0.020, p=0.983)$. The effect of 'problem type' remains significant after applying Tukey's range test correction for multiple comparisons $(p<0.05)$, which shows that there is a significant effect of problem type on prefrontal EEG asymmetry within one or more of the frequency bands of interest.

Inspecting asymmetry values in individual frequency bands between trials in which participants attempted a standard problem vs. trials in which they attempted a MM problem reveals a significant effect of problem type on observed prefrontal EEG asymmetry recorded from the first 30 s of the problem solving period in the alpha, beta, and gamma frequency bands (alpha band: $t(151)=-2.404, p=0.017$; beta 
band: $t(151)=-2.821, p=0.005$; gamma band: $t(151)=-2.409, p=0.017)$. Specifically, for trials in which participants attempted a MM problem a more positive prefrontal asymmetry was observed. This is illustrated in Figure 4.

Correction for multiple comparisons, via Holm-Bonferroni correction, revealed these effects to remain significant, after correction, at $p<0.05$, in all three frequency bands.

More positive prefrontal EEG asymmetry results from greater observed bandpower in the left hemisphere. Thus, our result indicates relatively greater left hemisphere neural activity. This suggests that while attempting to solve MM problems the bandpower of our participant's EEG was greater in the left hemisphere.

We also explored differences in EEG asymmetry on a per problem basis. Figure 5 illustrates the asymmetry in the alpha frequency band while participants attempted

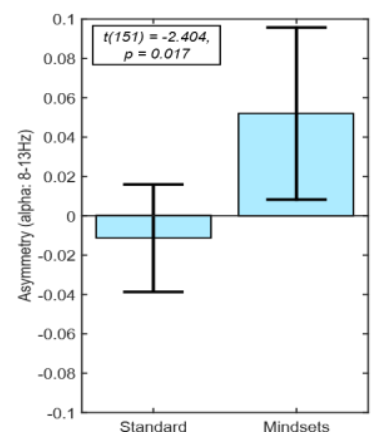

(a) Alpha

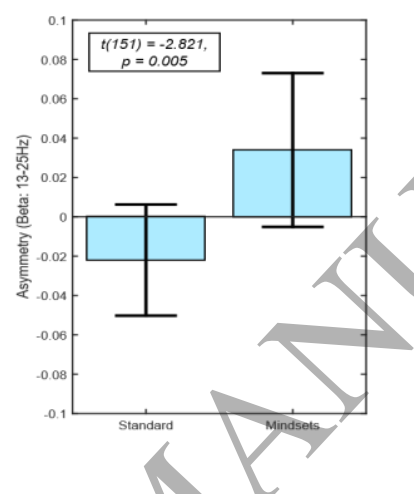

(b) Beta

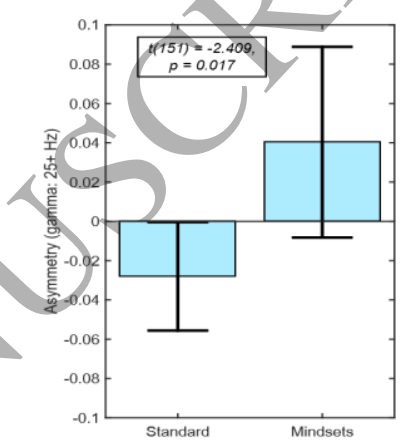

(c) Gamma

Figure 4: Mean prefrontal EEG asymmetry in the alpha, beta, and gamma frequency bands $(813 \mathrm{~Hz}, 13-$ $25 \mathrm{~Hz}$, and $25+\mathrm{Hz}$ ) observed while participants attempted standard problems and MM problems.

to solve each problem.

Although a significant difference $(p<0.05)$ was only observed for problem 2 (and this is not significant after Holm-Bonferroni multiple comparisons correction), a general trend of increasing asymmetry was observed for the majority of the problems. It may be the case that with a larger number of participants significant differences in asymmetry would also be observed for more of the individual problems.

Finally, motivated by recent evidence suggesting its involvement in affect and motivation [40,52], we also investigated mid-line theta EEG activity as a possible neural correlate of motivation. However, we did not find any significant differences $(t(151)=-0.865, p=0.388)$ between standard and MM formulated problems.

\subsection{Correlations between asymmetry and motivation}

We observe some significant correlations between EEG prefrontal asymmetry values and participants reports of their changes in motivation after attempting each problem. Specifically, we observed significant correlations in the alpha band when participants attempt to solve MM problems $(r(14)=0.291, p=0.019)$.

This result provides further evidence to reinforce the observation that the prefrontal EEG asymmetry is a neural correlate of motivation and, therefore may be used as an index of a participant's motivation as they attempt to solve a problem. 


\subsection{Task performance effects}

We investigated whether 'weaker' students (or 'stronger' students) find MM problems more motivating.

We did not find any significant correlations between student ability and changes in motivation (either from self-reports $(r(14)=0.155, p=0.630)$ or neural correlates of motivation $(r(13)=0.187, p=0.139))$. These results are from a very small sample size and, thus, it is not possible to draw a very strong conclusion from them. However, they do not suggest that 'weaker' students may benefit more from MM formulated problems in terms of change in motivation. Instead, our results suggest that MM formulated problems benefited all participants in our experiment equally.

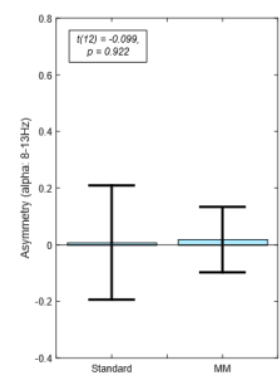

(a) Problem 1

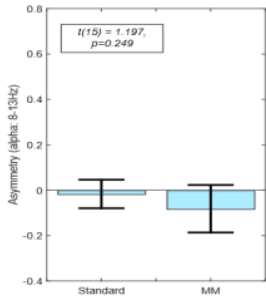

(f) Problem 6

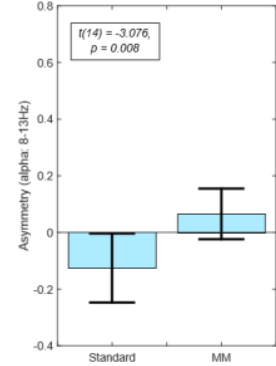

(b) Problem 2

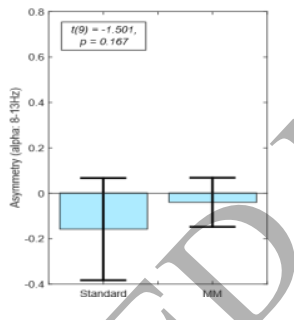

(g) Problem 7

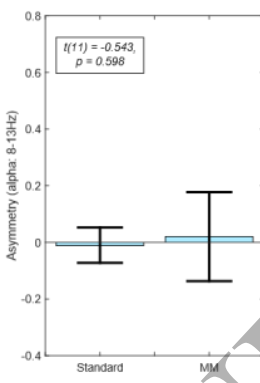

(c) Problem 3



(d) Problem 4

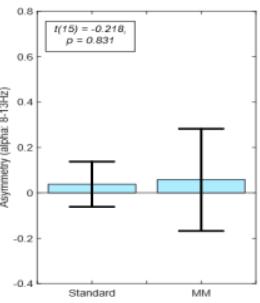

(i) Problem 9

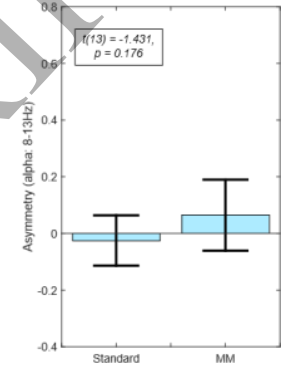

(e) Problem 5

Figure 5: Change in EEG asymmetry in the alpha frequency band $(8-13 \mathrm{~Hz})$ observed over all participants after attempting each type of problem (standard and MM problems) for each individual problem. Error bars indicate the confidence intervals. Significance testing was performed with a Student's $t$-test.

\section{Discussion}

Our results illustrate that the use of problems formulated as MM problems produce a significant increase in levels of motivation reported, while problems formulated in a standard way produce a decrease in reported motivation levels. Thus, our first hypothesis, that participants will report greater motivation when attempting MM problems, is validated. Consequently, this provides some of the first evidence that formulating problems as $\mathrm{MM}$ problems is less demotivating for students than presenting mathematical problems in a standard way.

Interestingly, this difference is apparent even though participants were not informed about MM theory and were unaware that some problems were formulated according to MM theory and others were not. This provides evidence that the use of MM theory to structure mathematical problems increases learner motivation even when learners are not explicitly aware of MM theory. 
It has been argued that standard mathematical problems are demotivating for some learners as failure to solve the problem reinforces negative self-images within the learner [48]. On the other hand, when problems are formulated as MM problems, failure to solve the problem may be seen by the student as a learning opportunity [48]. However, to date very little research has been undertaken to explore the brain processes underpinning this argument, and some of the evidence that does exist includes, according to a recent review [54] of the main book on mindset theory [11], 'numerous examples of an inappropriate use of neuroscience to back up educational claims in this book'. Our results go some way towards correcting this.

When considering individual problem types, it may be observed that the problem pairs in which participants reported larger increases in motivation when the problems were formulated according to MM theory (problems 2, 5, and 10) were constructed in one of two ways. Specifically, the MM problems of these pairs asked students to either apply a well-known formula implicitly rather than explicitly, or to consider points in space rather than algebraic formulas. It will be interesting to further study whether these two particular types of MM reformulations of problems are most effective at improving motivation. Note, however, that these changes in motivation for individual problems were not statistically significant after correction for multiple comparisons.

Therefore, these results should be interpreted with caution.

We also validate our second hypothesis, that neural responses to changes in motivation will differ significantly between attempts to solve MM problems and attempts to solve standard problems. The observation of greater EEG prefrontal asymmetry in the alpha and beta frequency bands while participants attempted to solve MM problems is interesting. Greater left prefrontal hemisphere activity (greater asymmetry) has been widely reported to correlate with greater levels of motivation $[53,19]$ and the approach-withdrawal response encapsulated in the "hemispheric valence hypothesis' $[23,34]$. Thus, the observed increase in this neural indicator suggests that participants were experiencing greater levels of motivation while attempting to solve problems of this type. This reinforces research from neuroscience that suggests an involvement of cortical and prefrontal regions in motivation [37].

It may be argued that the increased left-frontal asymmetry observed in the present study may be the result of increased valance in response to $\mathrm{MM}$ problems, as the viewing of positively valanced stimuli has also been evidenced to increase left-frontal asymmetry, as seen, for example, in work by Herrington and colleagues [35]. However, this is unlikely to be the case. Research, most notably by Berkman and Lieberman (2010), showed that although motivation and valance covary, they are distinct [7]. This has been evidenced in the present study, we found no significant difference in valance in participants ratings, however, we did find significant differences in reported motivation. This suggests that our results are likely to have occurred due to an increase in motivation and not an increase in valance.

It may also be argued that the difference in observed prefrontal EEG asymmetry values between MM and standard problems may simply be a result of differences in task difficulty between the two types of problem. Task difficulty has been reported to correlate with prefrontal EEG asymmetry [49], and our MM problems are observed to be more challenging for our participants. However, upon careful inspection of our results, we do not believe this to be the case for the following reasons. 
First, when our participants attempt to perform our MM problems, greater left hemisphere EEG bandpower is observed in the prefrontal cortex. This same pattern of a shift in prefrontal EEG towards the left hemisphere has been observed in a number of studies to correlate with increased motivation levels $[53,19]$. However, task difficulty has been reported to have the opposite effect, i.e. a move challenging task, with a greater associated mental workload, is known, from a small number of studies, to correlate with greater right hemisphere EEG bandpower in the prefrontal cortex [30, 33]. Furthermore, other effects that one may expect during a mathematical problem solving exercise, such as arithmetic learning, are known to involve cortical regions distinct from the pre-frontal cortex [56]. Thus, our EEG observations support the hypothesis that the MM problems are more motivating.

Second, our participants' self-reports of their levels of motivation indicate that they

found MM problems more motivating. This provides further evidence to support our hypothesis.

Task difficulty and motivation are not, of course, mutually exclusive, nor should one expect them to be perfectly negatively correlated. It is quite possible that some tasks may be both more difficult (challenging) and more motivating, while other tasks may be more difficult and less motivating. Our results suggest that the MM problems we use in this study are both more challenging for our participants and more motivating.

Additionally, our observation of significant correlations between prefrontal EEG asymmetry and participants' reports of their motivation further reinforces the observation that the change in EEG asymmetry is a result of a difference in motivation and not simply an effect of another process, such as different levels of workload. This provides further evidence in support of our conclusions.

Readers may be interested in individual and demographic differences in response to $\mathrm{MM}$ problems. We did investigate differences in participant ability, but did not find any significant differences. We regard our cohort of participants to be too small to robustly explore other demographic differences, such as gender.

In summary, MM problems are significantly more motivating than standard mathematical problems, as measured by self-reports and neural correlates of motivation. This provides some of the first evidence that $\mathrm{MM}$ theory improves motivation and the changes in neural activity that underpin this.

These results show that by presenting mathematical problems formulated according to MM theory to learners, it is possible to increase learner motivation. This happens by engaging the brains stimuli reward pathways (as evidenced by changes in prefrontal asymmetry in the EEG). Interestingly, this effect occurs even though participants were not explicitly made aware of MM theory. This provides evidence that, by only making simple changes to how mathematical problems are presented, it is possible to increase the motivation of mathematical learners.

\section{Acknowledgements}

The project was funded by the University of Essex through its Education Strategic Fund. 
References

[1] B. Aeschlimann, W. Herzog, and E. Makarova. How to foster students' motivation in mathematics and science classes and promote students' stem career choice. a study in swiss high schools. International Journal of Educational Research, 79:31 41, 2016.

[2] J. Aronson, C. B. Fried, and C. Good. Reducing the Effects of Stereotype Threat on African American College Students by Shaping Theories of Intelligence. Journal of Experimental Social Psychology, 38(2):113-125, 2002.

[3] M. Arsalidou, J. Pascual-Leone, J. Johnson, D. Morris, and M. J. Taylor. A balancing act of the brain: activations and deactivations driven by cognitive load. Brain and behavior, 3(3):273-85, 2013.

[4] D. Ary, L. Cheser Jacobs, C. K. Sorensen Irvine, and D. Walker. Introduction to Research in Education. Cengage, 2014.

[5] M. Attenborough. Engineering Mathematics Expósed. McGraw-Hill, 1994.

[6] J. Bahlmann, E. Aarts, and M. D'Esposito. Influence of Motivation on Control Hierarchy in the Human Frontal Cortex. Journal of Neuroscience, 35(7):32073217, 2015.

[7] E. T. Berkman and M. D. Lieberman. Approaching the Bad and Avoiding the Good: Lateral Prefrontal Cortical Asymmetry Distinguishes between Action and Valence. Journal of Cognitive Neuroscience, 22(9):1970-1979, 2010.

[8] R. Biehler. Didactics of mathematics as a scientific discipline. Kluwer Academic Publishers, 2002.

[9] J. Boaler. What's math got to do with it?: helping children learn to love their most hated subject-and why it's important for America. Penguin, 2008.

[10] J. Boaler. The elephant in the classroom: Helping children learn and love maths. Souvenir Press, 2015.

[11] J. Boaler. Mathematical mindsets: Unleashing students' potential through creative math, inspiring messages and innovative teaching. John Wiley \& Sons, 2015.

[12] J. Boaler and S. K. Selling. Psychological imprisonment or intellectual freedom? a longitudinal study of contrasting school mathematics approaches and their impact on adults' lives. Journal for Research in Mathematics Education, 48(1):78-105, 2017.

[13] M. Bruinsma. Motivation, cognitive processing and achievement in higher education. Learning and instruction, 14(6):549-568, 2004.

[14] B. Butterworth and V. Walsh. Neural basis of mathematical cognition. Current Biology, 21(16):R618-R621, 2011. 
[15] J. T. Cacioppo. Feelings and emotions: roles for electrophysiological markers. Biological psychology, 67(1-2):235-43, 2004.

[16] C. Chen and H. W. Stevenson. Motivation and mathematics achievement: A comparative study of asian-american, caucasian-american, and east asian high school students. Child development, 66(4):1215-1234, 1995.

[17] M. M. Chiu and Z. Xihua. Family and motivation effects on mathematics achievement: Analyses of students in 41 countries. Learning and Instruction, 18(4):321 - 336, 2008.

[18] J. A. Coan and J. J. Allen. Frontal EEG asymmetry as a moderator and mediator of emotion. Biological Psychology, 67(1-2):7-50, 2004.

[19] J. A. Coan and J. J. Allen. Frontal EEG asymmetry as a moderator and mediator of emotion. Biological Psychology, 67(1-2):7-50, 2004.

[20] A. Croft, T. Croft, and R. Davison. Mathematics for éngineers: a modern interactive approach. Pearson Education, 2008.

[21] I. Daly, A. Malik, F. Hwang, E. Roesch, J. Weaver, A. Kirke, D. Williams, E. Miranda, and S. J. Nasuto. Neural correlates of emotional responses to music: an EEG study. Neuroscience Letters, 573:52-57, 2014.

[22] I. Daly, R. Scherer, M. Billinger, and G. Muller-Putz. FORCe: Fully Online and automated artifact Removal for brain-Computer interfacing. IEEE transactions on neural systems and rehabilitation engineering, 23(5), 2014.

[23] R. Davidson. Cerebral asymmetry, emotion, and affective style. In R. Davidson and K. Hugdahl, editors, Brain asymmetry, pages 361-387. MIT Press, Cambridge, 1995.

[24] S. Dehaene, M. Piazza, P. Pinel, and L. Cohen. Three Parietal Circuits For Number Processing. Cognitive Neuropsychology, 20(3-6):487-506, 2003.

[25] S. Dettmers, U. Trautwein, O. Lüdtke, M. Kunter, and J. Baumert. Homework works if homework quality is high: Using multilevel modeling to predict the development of achievement in mathematics. Journal of Educational Psychology, 102(2):467-482, 2010.

[26] C. S. Dweck. Mindset : the new psychology of success. Random House, 2006.

[27] C. S. Dweck. Self-theories: Their role in motivation, personality, and development. psychology press, 2013.

[28] G. L. Dweck, Carol S.|Walton, Gregory M.|Cohen. Academic Tenacity: Mindsets and Skills that Promote Long-Term Learning. Bill \& Melinda Gates Foundation, 2014. 
[29] J. B. Earle. Task Difficulty and EEG Alpha Asymmetry: An Amplitude and Frequency Analysis. Neuropsychobiology, 20(2):96-112, 1988.

[30] D. Galin, J. Johnstone, and J. Herron. Effects of task difficulty on EEG measures of cerebral engagement. Neuropsychologia, 16(4):461-472, 1978.

[31] H. Gaspard, A.-L. Dicke, B. Flunger, B. M. Brisson, I. Häfner, B. Nagengast, and U. Trautwein. Fostering adolescents' value beliefs for mathematics with a relevance intervention in the classroom. Developmental Psychology, 51(9):1226-1240, 2015.

[32] H. Grant and C. S. Dweck. Clarifying Achievement Goals and Their Impact. Journal of Personality and Social Psychology, 85(3):541-553, 2003.

[33] E. Harmon-Jones, L. Y. Abramson, R. Nusslock, J. D. Sigelman, S. Urosevic, L. D. Turonie, L. B. Alloy, and M. Fearn. Effect of Bipolar Disorder on Left Frontal Cortical Responses to Goals Differing in Valence and Task Difficulty. Biological Psychiatry, 63(7):693-698, 2008.

[34] K. M. Heilman. The neurobiology of emotional experience. The Journal of Neuropsychiatry and Clinical Neurosciences, 9(3):439-448, 1997.

[35] J. D. Herrington, A. Mohanty, N. S. Koven, J. E. Fisher, J. L. Stewart, M. T. Banich, A. G. Webb, G. A. Miller, and W. Heller. Emotion-Modulated Performance and Activity in Left Dorsolateral Prefrontal Cortex. Emotion, 5(2):200- 207, 2005.

[36] B. K. Hofer. Instructional context in the college mathematics classroom: Epistemological beliefs and student motivation. Journal of Staff, Program \& Organization Development, 16(2):73-82, 1999.

[37] C. B. Holroyd and N. Yeung. Motivation of extended behaviors by anterior cingulate cortex. Trends in Cognitive Sciences, 16(2):122-128, 2012.

[38] T. N. Hopfenbeck and M. Kjærnsli. Students' test motivation in PISA: the case of Norway. The Curriculum Journal, 27(3):406-422, 2016.

[39] A. Ischebeck, L. Zamarian, C. Siedentopf, F. Koppelstätter, T. Benke, S. Felber, and M. Delazer. How specifically do we learn? Imaging the learning of multiplication and subtraction. Neurolmage, 30(4):1365-1375, 2006.

[40] R. Ishii, L. Canuet, T. Ishihara, Y. Aoki, S. Ikeda, M. Hata, T. Katsimichas, A. Gunji, H. Takahashi, T. Nakahachi, M. Iwase, and M. Takeda. Frontal midline theta rhythm and gamma power changes during focused attention on mental calculation: an MEG beamformer analysis. Frontiers in human neuroscience, 8:406, 2014.

[41] R. C. Kadosh and V. Walsh. Numerical representation in the parietal lobes: Abstract or not abstract? Behavioral and Brain Sciences, 32(3-4):313, 2009.

[42] S. Koelsch. Brain correlates of music-evoked emotions. Nature Reviews Neuroscience, 15(3):170-180, 2014. 
[43] R. A. Lazowski and C. S. Hulleman. Motivation Interventions in Education: A MetaAnalytic Review. Review of Educational Research, 86(2):602-640, 2016.

[44] J. A. Middleton and P. A. Spanias. Motivation for achievement in mathematics: Findings, generalizations, and criticisms of the research. Journal for research in Mathematics Education, pages 65-88, 1999.

[45] J. Morris. Observations: SAM: The Self-Assessment Manikin; An Efficient CrossCultural Measurement of Emotional Response. Journal of Advertising Research, 35(8), 1995.

[46] J. S. Moser, H. S. Schroder, C. Heeter, T. P. Moran, and Y.-H. Lee. Mind Your Errors. Psychological Science, 22(12):1484-1489, 2011.

[47] M. M. Müller, A. Keil, T. Gruber, and T. Elbert. Processing of affective pictures modulates right-hemispheric gamma band EEG activity. Clinical neurophysiology : official journal of the International Federation of Clinical Neurophysiology, 110(11):1913-20, 1999.

[48] B. Ng and Betsy. The Neuroscience of Growth Mindset and Intrinsic Motivation. Brain Sciences, 8(2):20, 2018.

[49] M. Osaka. Peak Alpha Frequency of EEG during a Mental Task: Task Difficulty and Hemispheric Differences. Psychophysiology, 21(1):101-105, 1984.

[50] P. R. Pintrich. The role of motivation in promoting and sustaining self-regulated learning. International Journal of Educational Research, 31(6):459-470, 1999.

[51] G. R. Price, I. Holloway, P. Räsänen, M. Vesterinen, and D. Ansari. Impaired parietal magnitude processing in developmental dyscalculia. Current Biology, 17(24):R1042-R1043, 2007.

[52] D. Sammler, M. Grigutsch, T. Fritz, and S. Koelsch. Music and emotion: electrophysiological correlates of the processing of pleasant and unpleasant music. Psychophysiology, 44(2):293-304, 2007.

[53] L. A. Schmidt and L. J. Trainor. Frontal brain electrical activity (EEG) distinguishes valence and intensity of musical emotions. Cognition \& Emotion, 15(4):487-500, 2001.

[54] V. Simms. Mathematical mindsets: unleashing students' potential through creative math, inspiring messages and innovative teaching. Research in Mathematics Education, 18(3):317-320, 2016.

[55] K. Singh, M. Granville, and S. Dika. Mathematics and science achievement: Effects of motivation, interest, and academic engagement. The Journal of Educational Research, 95(6):323-332, 2002. 
[56] M. Soltanlou, C. Artemenko, A.-C. Ehlis, S. Huber, A. J. Fallgatter, T. Dresler, and H.-C. Nuerk. Reduction but no shift in brain activation after arithmetic learning in children: A simultaneous fNIRS-EEG study. Scientific Reports, 8(1):1707, 2018.

[57] J. M. Spielberg, J. L. Stewart, R. L. Levin, G. A. Miller, and W. Heller. Prefrontal Cortex, Emotion, and Approach/Withdrawal Motivation. Social and personality psychology compass, 2(1):135-153, 2008.

[58] T. Stevens, A. Olivarez, W. Y. Lan, and M. K. Tallent-Runnels. Role of mathematics self-efficacy and motivation in mathematics performance across ethnicity. The Journal of Educational Research, 97(4):208-222, 2004.

[59] D. Stipek, J. M. Salmon, K. B. Givvin, E. Kazemi, G. Saxe, and V. L. MacGyvers. The value (and convergence) of practices suggested by motivation research and promoted by mathematics education reformers. Journal for Research in mathematics education, pages 465-488, 1998.

[60] P. Terenzini, E. Pascarella, and G. Blimling. Students' Out-of-Class Experiences and Their Influence on Learning and Cognitive Development: A Literature Review. Journal of College Student Development, 37(2):149-162, 1996.

[61] V. Trowler. Student engagement literature review | Higher Education Academy. Technical report, 2010.

[62] C. D. Tsang, L. J. Trainor, D. L. Santesso, S. L. Tasker, and L. A. Schmidt. Frontal EEG responses as a function of affective musical features. Annals of the New York Academy of Sciences, 930:439-42, 2001.

[63] K. Wæge. Motivation for learning mathematics in terms of needs and goals. In Proceedings of CERME, volume 6, pages 84-93, 2009.

[64] M. Walpulski. EEG representation of emotion evoking pictures. 2008.

[65] H. M. Watt, J. D. Shapka, Z. A. Morris, A. M. Durik, D. P. Keating, and J. S. Eccles. Gendered motivational processes affecting high school mathematics participation, educational aspirations, and career plans: A comparison of samples from australia, canada, and the united states. Developmental psychology, 48(6):1594, 2012.

[66] L. Zago, M. Pesenti, E. Mellet, F. Crivello, B. Mazoyer, and N. TzourioMazoyer. Neural Correlates of Simple and Complex Mental Calculation. Neurolmage, 13(2):314-327, 2001. 


\section{Appendices}

\section{A: The problems used}

S1 Find the derivative of the function $2 x^{2}+3 x+4$.

M1 Combine all symbols below into a function whose derivative is $4 x+3$. Symbols to use are: $x, x^{2}, 2,3,4,+,+$.

S2 Find $x$ and $y$ by solving simultaneous equations $\begin{gathered}x+5 y=23 \\ x+10 y=33\end{gathered}$

M2 Assuming that $x+5 y=23$ and $x+10 y=33$, find $x+y$.

S3 Consider two vectors $\mathbf{u}=(1,3)$ and $\mathbf{v}=(-3,-5)$. Find $\frac{1}{2}(\mathbf{u}+\mathbf{v})$.

M3 Consider a straight line segment whose endpoints have coordinates $(1,3)$ and $(-3,-5)$. Find the coordinates of the centre of the segment.

$\mathrm{S} 4$ Consider a differential equation $\frac{\mathrm{d} y}{\mathrm{~d} x}=2 y$. One can rearrange it as $\frac{\mathrm{d} y}{y}=2 \mathrm{~d} x$, then integrate $\int \frac{\mathrm{d} y}{y}=\int 2 \mathrm{~d} x$, producing $\ln y=2 x$. Solve the last equation for $y$ to find a function which is a solution of the differential equation $\frac{\mathrm{d} y}{\mathrm{~d} x}=2 y$.

M4 The function $f(x)=e^{x}$ is a solution of the differential equation $f^{\prime}=f$. Using this example and changing it as needed, find a function which is a solution of the differential equation $f^{\prime}=2 f$.

S5 The modulus of a complex number $a+b i$ is defined as $\sqrt{a^{2}+b^{2}}$. Find the modulus of the complex number $1+i$.

M5 The modulus of a complex number $a+b i$ is defined as the distance between the origin $(0,0)$ and the point with coordinates $(a, b)$. Find the modulus of the complex number $1+i$.

S6 Find the derivative of the function $f(x)=x^{3}-x$. Consider the value of the derivative at the point $x=0$ and decide whether the function $f(x)$ at the point $x=0$

- has a positive gradient, or

- has a negative gradient, or

- is horizontal.

M6 Consider the function $f(x)=x^{3}-x$. Which one of the following is correct? At the point $x=0$, the function $f(x)$

- has a positive gradient, or

has a negative gradient, or 
- is horizontal.

S7 The general solution of the differential equation $f^{\prime}=2$ is $f(x)=2 x+C$. By choosing an approriate value of $C$, find a solution passing through the point $x=0, y=1$.

M7 Find a function $f(x)$ which is a solution of the differential equation $f^{\prime}=2$ and passes through the point $x=0, y=1$.

S8 A straight line on the plane can be represented by an equation of the form $a x+b x=c$, where $a, b, c$ are some numbers. Find an equation of the straight line passing through the points $(0,1)$ and $(2,0)$. Hint: the line passing through two points $\left(x_{1}, y_{1}\right)$ and $\left(x_{2}, y_{2}\right)$ can be represented by an equation

$$
\frac{y-y_{1}}{x-x_{1}}=\frac{y_{2}-y_{1}}{x_{2}-x_{1}}
$$

M8 A straight line on the plane can be represented by an equation of the form $a x+b x=c$, where $a, b, c$ are some numbers. Find an equation of the straight line passing through the points $(0,1)$ and $(2,0)$.

S9 Calculate the number of ways of choosing three objects from 10 distinct objects. Use the formula

$$
\left(\begin{array}{c}
n \\
r
\end{array}\right)=\frac{n !}{r !(n-r) !}
$$

M9 Calculate the number of ways of choosing three objects from 10 distinct objects. S10 Find the product of matrices

$$
\left(\begin{array}{ll}
0 & 1 \\
1 & 0
\end{array}\right)\left(\begin{array}{ll}
a & b \\
c & d
\end{array}\right)
$$

M10 What will happen to the matrix $\left(\begin{array}{ll}a & b \\ c & d\end{array}\right)$ if you multiply it by the matrix $\left(\begin{array}{ll}0 & 1 \\ 1 & 0\end{array}\right) ?$ Will

- the rows be swapped, or

- the columns be swapped?

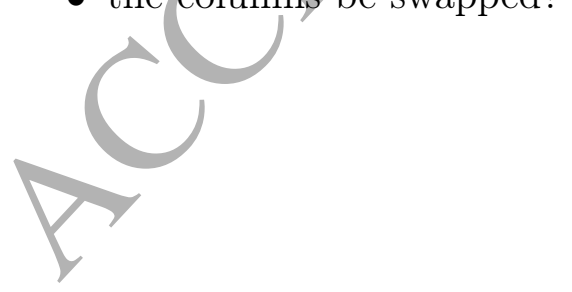

